\title{
A Relativistic Symmetry in Nuclei
}

\author{
Joseph N. Ginocchio \\ Theoretical Division, Los Alamos National Laboratory, Los Alamos, New Mexico 87545, USA
}

\begin{abstract}
We review the status of a quasi - degenerate doublets in nuclei, called pseudospin doublets, which were discovered about thirty years ago and the origins of which have remained a mystery, until recently. We show that pseudospin doublets originate from an SU(2) symmetry of the Dirac Hamiltonian which occurs when the scalar and vector potentials are opposite in sign but equal in magnitude. Furthermore, we survey the evidence that pseudospin symmetry is approximately conserved for a Dirac Hamiltonian with realistic scalar and vector potentials. We briefly discuss the relationship of pseudospin symmetry with chiral symmetry and the implications of pseudospin symmetry for the antinucleon spectrum in nuclei.
\end{abstract}

Typeset using REVTEX 


\section{INTRODUCTION}

Dick Slansky and I met almost thirty years ago as assistant professors at Yale University. Although,for the most part, Dick's published research at that time was in hadron collisions [1], he became interested in group theory and symmetry, particularly applied to elementary particles physics, during his stay at Yale. I had similar interest in group theory and symmetry, but primarily applied to the many - body problem, particularly in nuclear physics. In the mid seventies Dick and I each accepted positions at Los Alamos National Laboratory and we continued to discuss symmetry in physics. At Los Alamos he published a number of papers in group theory including his very popular review on "Group Theory for Unified Model Building" [2], and also published a book on affine Lie algebras [3]. In 1989 he became Theoretical Division Leader at the Los Alamos National Laboratory, and, technically, my "boss".

At the time we were at Yale I had heard about an observation that certain single nucleon levels in spherical nuclei were found to clump into quasi - degenerate doublets 田,5]. The term pseudospin doublets was applied to these states, although the quasi - degeneracies we assumed to be accidental. About two years ago I rexamined these pseudospin doublets, the origin of which remained a mystery, and I discovered that they are a consequence of a relativistic symmetry [6,7]. I discussed this revelation with Dick and he suggested that this symmetry may have some connection with chiral symmetry. In this paper I would like to discuss the progress that has been made in understanding pseudospin symmetry, including an enticing connection with chiral symmetry via QCD sum rules. 


\section{PSEUDOSPIN SYMMETRY}

The spherical shell model orbitals that were observed to be quasi - degenerate have non relativistic quantum numbers $\left(n_{r}, \ell, j=\ell+1 / 2\right)$ and $\left(n_{r}-1, \ell+2, j=\ell+3 / 2\right)$ where $n_{r}, \ell$, and $j$ are the single-nucleon radial, orbital, and total angular momentum quantum numbers, respectively [4,5]. This doublet structure is expressed in terms of a "pseudo" orbital angular momentum $\tilde{\ell}=\ell+1$, the average of the orbital angular momentum of the two states in doublet and "pseudo" spin, $\tilde{s}=1 / 2$. For example, $\left(n_{r} s_{1 / 2},\left(n_{r}-1\right) d_{3 / 2}\right)$ will have $\tilde{\ell}=1$, $\left(n_{r} p_{3 / 2},\left(n_{r}-1\right) f_{5 / 2}\right)$ will have $\tilde{\ell}=2$, etc. These doublets are almost degenerate with respect

to pseudospin, since $j=\tilde{\ell} \pm \tilde{s}$ for the two states in the doublet; examples are shown in Figure 1. Pseudospin "symmetry" was shown to exist in deformed nuclei as well [8,9] and has been used to explain features of deformed nuclei, including superdeformation 10 and identical bands [11,12]. However, the origin of pseudospin symmetry remained a mystery and "no deeper understanding of the origin of these (approximate) degeneracies" existed [13. A few years ago it was shown that relativistic mean field theories gave approximately the correct spin orbit splitting to produce the pseudospin doublets [14. In this paper we shall review more recent developements that show that pseudospin symmetry is a relativistic symmetry 6, $6,15,7$.

\section{SYMMETRIES OF THE DIRAC HAMILTONIAN}

The success of the shell model implies that nucleons move in a mean field produced by the interactions between the nucleons. Normally, it suffices to use the Schrodinger equation to describe the motion of the nucleons in this mean field. However, in order to understand the origin of pseudospin symmetry, we need to take into account the motion of the nucleons in a relativistic mean field and thus use the Dirac equation. The Dirac Hamiltonian, H, with 
an external scalar, $V_{S}$, and vector, $V_{V}$, potentials is given by:

$$
H=\vec{\alpha} \cdot \vec{p}+\beta\left(m+V_{S}\right)+V_{V}
$$

where we have set $\hbar=c=1, \vec{\alpha}, \beta$ are the usual Dirac matrices [16], $m$ is the nucleon mass, and $\vec{p}$ is the three momentum. The Dirac Hamiltonian is invariant under an $\mathrm{SU}(2)$ algebra for two limits: $V_{S}-V_{V}=$ constant and $V_{S}+V_{V}=$ constant. For finite nuclei the constant will be zero since each potential will go to zero at large distances [17]. (For infinite nuclear matter the constant could be non-zero.)

The generators for the $\mathrm{SU}(2)$ algebra, $\hat{S}_{i}$, which commute with the Dirac Hamiltonian, $\left[H, \hat{S}_{i}\right]=0$, for the case when $V_{S}=V_{V}$ are given by [17

$$
\hat{S}_{i}=\frac{\vec{\alpha} \cdot \vec{p} \hat{s}_{i} \vec{\alpha} \cdot \vec{p}}{p^{2}} \frac{(1-\beta)}{2}+\hat{s}_{i} \frac{(1+\beta)}{2}
$$

where $\hat{s}_{i}=\sigma_{i} / 2$ are the usual spin generators and $\sigma_{i}$ the Pauli matrices. This reduces to

$$
\hat{S}_{i}=\left(\begin{array}{cc}
\hat{s}_{i} & 0 \\
0 & \hat{\tilde{s}}_{i}
\end{array}\right)
$$

where

$$
\hat{\tilde{s}}_{i}=U_{p} \hat{s}_{i} U_{p}=\frac{2 \vec{s} \cdot \vec{p}}{p^{2}} p_{i}-\hat{s}_{i}
$$

In (四) $U_{p}=\frac{\vec{\sigma} \cdot \vec{p}}{p}$ is the momentum-helicity unitary operator introduced in 14 that accomplishes the transformation from the normal shell model space to the pseudo shell model space while preserving rotational, parity, time - reversal, and translational invariance. This symmetry limit leads to spin doublets, but we know that the spin orbit splitting in nuclei is very large so this limit is not applicable to nuclei. However we shall see that it is relevant for the antinucleon spectrum.

We shall show that, in the limit of $V_{S}=-V_{V}$, the conserved symmetry is pseudospin symmetry. The generators for the $\mathrm{SU}(2)$ algebra, $\hat{\widetilde{S}}_{i}$, which commute with the Dirac Hamiltonian, $\left[H, \hat{\tilde{S}}_{i}\right]=0$, for the case when $V_{S}=-V_{V}$ are given by [17] 


$$
\hat{\tilde{S}}_{i}=\frac{\vec{\alpha} \cdot \vec{p} \hat{s}_{i} \vec{\alpha} \cdot \vec{p}}{p^{2}} \frac{(1+\beta)}{2}+\hat{s}_{i} \frac{(1-\beta)}{2} .
$$

This reduces to

$$
\hat{\widetilde{S}}_{i}=\left(\begin{array}{cc}
\hat{\tilde{s}}_{i} & 0 \\
0 & \hat{s}_{i}
\end{array}\right)
$$

For $V_{S}=-V_{V}$, the eigenfunctions of the Dirac Hamiltonian, $H \Psi_{\tau, \tilde{\mu}}=\mathcal{E}_{\tau} \Psi_{\tau, \tilde{\mu}}$ are doublets $(\tilde{S}=1 / 2, \tilde{\mu}= \pm 1 / 2)$ with respect to the $\mathrm{SU}(2)$ generators $\hat{\widetilde{S}}_{i}$ of Eq. (6)

$$
\begin{aligned}
\hat{\tilde{S}}_{z} \Psi_{\tau, \tilde{\mu}} & =\tilde{\mu} \Psi_{\tau, \tilde{\mu}} \\
\hat{\tilde{S}}_{ \pm} \Psi_{\tau, \tilde{\mu}} & =\sqrt{(1 / 2 \mp \tilde{\mu})(3 / 2 \pm \tilde{\mu})} \Psi_{\tau, \tilde{\mu} \pm 1}, \quad\left(V_{S}=-V_{V}\right)
\end{aligned}
$$

where $\hat{\widetilde{S}}_{ \pm}=\hat{\widetilde{S}}_{x} \pm i \hat{\widetilde{S}}_{y}$. The eigenvalue $\tau$ refer to the other necessary quantum numbers.

The fact that the the pseudo - spin generators (6) have only the spin operator $\hat{s}_{i}$ operating on the lower component of the Dirac wave function has the consequence that the spatial wavefunctions for the two states in the pseudospin doublet are identical in the limit of $V_{S}=-V_{V}$ to within an overall phase.

This symmetry for $V_{S}=-V_{V}$ is general and applies to deformed nuclei as well as spherical nuclei. In the case for which the potentials are spherically symmetric, the Dirac Hamiltonian has an additional invariant SU(2) algebra; namely, the pseudo - orbital angular momentum,

$$
\hat{\tilde{L}}_{i}=\left(\begin{array}{cc}
\hat{\tilde{\ell}}_{i} & 0 \\
0 & \hat{\ell}_{i}
\end{array}\right)
$$

where $\hat{\tilde{\ell}}_{i}=U_{p} \hat{\ell}_{i} U_{p}, \hat{\ell}_{i}=\vec{r} \times \vec{p}$. In this limit, the Dirac wave functions are eigenfunctions of the Casimir operator of this algebra, $\hat{\tilde{L}} \cdot \hat{\tilde{L}}\left|\Psi_{\tau, \tilde{\ell}, j, m_{j}}\right\rangle=\tilde{\ell}(\tilde{\ell}+1)\left|\Psi_{\tau, \tilde{\ell}, j, m_{j}}\right\rangle$, where we have used a coupled basis, and $j$ is the eigenvalue of the total angular momentum operator $\hat{J}_{i}=\hat{\tilde{L}}_{i}+\hat{\tilde{S}}_{i}, \hat{J} \cdot \hat{J}\left|\Psi_{\tau, \tilde{\ell}, j, m_{j}}\right\rangle=j(j+1)\left|\Psi_{\tau, \tilde{\ell}, j, m_{j}}\right\rangle$, and $m_{j}$ is the eigenvalue of $\hat{J}_{z}$. Thus pseudo-orbital angular momentum as well as pseudospin are conserved in the spherical limit and $V_{S}=-V_{V}$. From (8), we see that the lower component wave function will have spherical 
harmonic of rank $\tilde{\ell}$ coupled to spin to give total angular momentum $j$. Since $\vec{\sigma} \cdot \vec{p}$ conserves the total angular momentum but $\vec{p}$ changes the orbital angular momentum by one unit because of parity conservation, the upper component also has total angular momentum $j$, but orbital angular momentum $\ell=\tilde{\ell} \pm 1$. If $j=\tilde{\ell}+1 / 2$, then it follows that $\ell=\tilde{\ell}+1$, whereas if $j=\tilde{\ell}-1 / 2$, then $\ell=\tilde{\ell}-1$. This agrees with the pseudospin doublets originally observed [4, [0] and discussed at the beginning of this paper.

For axially symmetric deformed nuclei, there is a $\mathrm{U}(1)$ generator corresponding to the pseudo-orbital angular momentum projection along the symmetry axis which is conserved in addition to the pseudospin for $V_{S}=-V_{V}$,

$$
\hat{\tilde{\lambda}}=\left(\begin{array}{cc}
\hat{\tilde{\Lambda}} & 0 \\
0 & \hat{\Lambda}
\end{array}\right),
$$

where $\hat{\tilde{\Lambda}}=U_{p} \hat{\Lambda} U_{p}$. In this case the Dirac wave functions are eigenfunctions of $\hat{\tilde{\lambda}}, \hat{\tilde{\lambda}}\left|\Psi_{\tau, \tilde{\Lambda}, \Omega}\right\rangle=$ $\tilde{\Lambda}\left|\Psi_{\tau, \tilde{\Lambda}, \Omega}\right\rangle$, where $\Omega$ is total angular momentum projection, $\Omega=\tilde{\Lambda}+\tilde{\mu}$, which has the same value for the upper and lower components since $\vec{\sigma} \cdot \vec{p}$ conserves the total angular momentum projection. Thus $\Omega=\tilde{\Lambda} \pm 1 / 2$, corresponding exactly to the quantum numbers of the pseudospin doublets for axially deformed nuclei discussed in [8,9].

However, the exact symmetry limit can not be realized in nuclei, because, if $V_{S}=-V_{V}$, there are no Dirac bound valence states and hence nuclei can not exist. However, we now show that pseudospin symmetry is approximately realized for $V_{S} \approx-V_{V}$.

\section{FREE NUCLEONS}

For a free Dirac nucleon, $V_{S}=V_{V}=0$, and hence both symmetries are valid. The free nucleon wavefunction is

$$
\Psi_{p, \mu}=\mathcal{N}\left(\begin{array}{c}
u_{\mu} \\
\frac{2 \mu p_{z} u_{\mu}}{\left(m+E_{p}\right)}
\end{array}\right) e^{i\left(p_{z} z-E_{p} t\right)}
$$


where $u_{\mu}$ are the Dirac spinors $u_{1 / 2}=\left(\begin{array}{l}1 \\ 0\end{array}\right), u_{-1 / 2}=\left(\begin{array}{l}0 \\ 1\end{array}\right), E_{p}=\sqrt{p^{2}+m^{2}}$, and $\mathcal{N}$ is the normalization. This wavefunction has definite helicity and hence is an eigenfunction of the helicity operator,

$$
U_{p} \Psi_{p, \mu}=2 \mu \Psi_{p, \mu}
$$

The wavefunctions with $\mu= \pm 1 / 2$ are doublets for $\hat{S}_{i}$ and, since $\hat{\tilde{S}}_{i}=U_{p} \hat{S}_{i} U_{p}$, the wavefunctions $\tilde{\Psi}_{p, \tilde{\mu}}=U_{p} \Psi_{p, \mu}=2 \mu \Psi_{p, \mu}$ are doublets for $\tilde{\widetilde{S}}_{i}$, the pseudospin.

\section{REALISTIC MEAN FIELDS}

A near equality in the magnitude of mean fields, $V_{S} \approx-V_{V}$, is a universal feature of the relativistic mean field approximation (RMA) of relativistic field theories with interacting nucleons and mesons [18 and relativistic theories with nucleons interacting via zero range interactions [19], as well as a consequence of QCD sum rules [20]. We shall discuss QCD sum rules in the next section.

Recently realistic relativistic mean fields were shown to exhibit approximate pseudospin symmetry in both the energy spectra and wave functions [15,21,22. In Fig. 2 we show the

energy splittings between pseudospin doublets normalised by $2 \tilde{\ell}+1$ as a function of the average binding energy $\langle\epsilon\rangle=\left(\epsilon_{\tilde{\ell}+1 / 2}+\epsilon_{\tilde{\ell}-1 / 2}\right) / 2$. We see that the energy splitting for the same pseudo - orbital angular momentum decreases as the radial quantum increases; that is, as the binding energy decreases. Also for the same binding energy, the energy splitting increases as the pseudo - orbital angular momentum increases. These features follow from the square well potential [6]. In Table 1 we tabulate some of the calculated energy splittings compared to the measured splittings. What is interesting is that the measured splittings are smaller than those calculated by relativistic mean field theory indicating that pseudospin symmetry breaking is overestimated by the relativistic mean field approximation. 
Pseudospin doublets will manifest themselves for deformed potentials as well when $V_{S} \approx$ $-V_{V}$. In Figure 3 the single particle (s.p.) energies of the doublets are plotted versus the deformation. We see that for each value of pseudo asymptotic quantum $\left[\tilde{N} \tilde{n}_{3} \tilde{\Lambda}\right]$ numbers there is a quasi-degenerate pseudospin doublet with $\Omega=\tilde{\Lambda} \pm 1 / 2$.

As mentioned in the last section the relativistic SU(2) pseudo-spin symmetry implies that the spatial wavefunction for the lower component of the Dirac wavefunctions will be equal in shape and magnitude for the two states in the doublet. For spherical nuclei the Dirac wavefunction for the two states in the doublet $\quad$ are $\quad \Psi_{\tau, j=\tilde{\ell}+1 / 2, m}=\left(g_{\tau, \tilde{\ell}+1 / 2}\left[Y_{\tilde{\ell}+1} \chi\right]_{m}^{j=\tilde{\ell}+1 / 2}, i f_{\tau, \tilde{\ell}+1 / 2}\left[Y_{\tilde{\ell}} \chi\right]_{m}^{j=\tilde{\ell}+1 / 2}\right), \quad \Psi_{\tau, j=\tilde{\ell}-1 / 2, m}=$ $\left(g_{\tau, \tilde{\ell}-1 / 2}\left[Y_{\tilde{\ell}-1} \chi\right]_{m}^{(j=\tilde{\ell}-1 / 2)}, i f_{\tau, \tilde{\ell}-1 / 2}\left[Y_{\tilde{\ell}} \chi\right]_{m}^{(j=\tilde{\ell}-1 / 2)}\right)$ where $g, f$ are the radial wave functions, $Y_{\ell}$ are the spherical harmonics, $\chi$ is a two-component Pauli spinor, and $[\ldots]^{(j)}$ means coupled to angular momentum $j$. For a square well potential, the overall phase between the two amplitudes will be a minus sign [6] so we expect that, in the symmetry limit for realistic potentials, $f_{\tau, \tilde{\ell}+1 / 2}(r)=-f_{\tau, \tilde{\ell}-1 / 2}(r)$. In Figure 4 we see that, for realistic zero range potentials, $f_{\tau, \tilde{\ell}+1 / 2}(r) \approx-f_{\tau, \tilde{\ell}-1 / 2}(r)[15$.

These results are also valid for the relativistic mean field approximation to a nuclear field theory with meson exchanges [21]. In Figure 5a) the pseudospin doublets in the vicinity of the Fermi surface for neutrons and protons are shown. The upper (g) and lower components (f) of the pseudospin doublets are also shown in Figure 5b,c,d. While the upper components are very different with different nodal stucture, the lower components are almost identical. We also note that the lower components are small with respect to the upper components, which is consistent with the non-relativistic shell model. 


\section{QCD SUM RULES}

Applying QCD sum rules in nuclear matter, the scalar and vector self-energies were determined to be [20]

$$
\begin{aligned}
& \Sigma_{s}=-\frac{4 \pi^{2} \sigma_{N} \rho_{N}}{m^{2} m_{q}} \\
& \Sigma_{v}=\frac{32 \pi^{2} \rho_{N}}{m^{2}}
\end{aligned}
$$

where $\rho_{N}$ is the nuclear density, and $m_{q}$ the quark mass. $\sigma_{N}$ is the sigma term which arises from the breaking of chiral symmetry [23]. The ratio then becomes

$$
\frac{\Sigma_{s}}{\Sigma_{v}}=-\frac{\sigma_{N}}{8 m_{q}}
$$

For reasonable values of $\sigma_{N}$ and quark masses, this ratio is close to -1 . The implication of these results is that chiral symmetry breaking is responsible for the scalar field being approximately equal in magnitude to the vector field, thereby producing pseudospin symmetry.

\section{ANTINUCLEON SPECTRUM}

The antinucleon states are obtained by charge conjugation, $C$, applied to the negative energy eigenstates of the Dirac Hamiltonian [16]. This leads to a spectrum which has quasi

- degenerate spin doublets, not pseudospin doublets. This follows from the fact that, under charge conjugation,

$$
C^{\dagger} \hat{\tilde{S}}_{i}, C=\left(\begin{array}{cc}
\hat{s}_{i} & 0 \\
0 & \hat{\tilde{s}}_{i}
\end{array}\right)=\hat{S}_{i}
$$

Thus in (14) the spin operator $\hat{s}_{i}$ operates on the upper component and hence the spatial wavefunctions for the upper components of the states in spin doublet will be very similar. Likewise for spherical nuclei, the pseudo-orbital angular momentum goes into the orbital 
angular momentum, and for axially deformed nuclei, pseudo-orbital projection goes into orbital projection along the body fixed z axis.

This symmetry in the antinucleon spectrum also follows from the fact that the antinucleon potentials are $\bar{V}_{S}=C^{\dagger} V_{S} C=V_{S}$, and $\bar{V}_{V}=C^{\dagger} V_{V} C=-V_{V}$. Thus $\bar{V}_{S} \approx \bar{V}_{V}$ and the symmetry of the Dirac Hamiltonian generated by (3) applies and spin doublets are produced in the antinucleon spectrum [17].

\section{SUMMARY}

We have shown that pseudospin symmetry is a broken $\mathrm{SU}(2)$ symmetry of the Dirac Hamilonian which describes the motion of nucleons in realistic scalar and vector mean field potentials, $V_{S} \approx-V_{V}$. This symmetry predicts that the spatial wavefunctions of the lower components for states in the doublet will be very similar in shape and size and this has been substantiated by relativistic mean field approximations of relativistic nuclear field theories and relativistic nuclear Lagrangians with zero range interactions. This symmetry has been linked via QCD sum rules to chiral symmetry breaking in nuclei. Finally, the antinucleon spectrum is shown to have a spin symmetry rather than a pseudospin symmetry.

Future applications of pseudospin symmetry will involve the testing of the wavefunction through the relationships between transitions within pseudospin doublets which follow from pseudospin symmetry [24].

\section{ACKNOWLEGEMENTS}

This work was supported by the United States Department of Energy under contract W-7405-ENG-36. 


\section{REFERENCES}

[1] Slansky R 1974 Phys. Rep. 1199

[2] Slansky R 1981 Phys. Rep. 791

[3] Kass S, Moody R V, Patera J, and Slansky R 1990 Affine Lie Algebras, Weight Multiplicities, and Branching Rules (Berkeley: University of California Press)

[4] Hecht K T and Adler A 1969 Nucl. Phys. A137 129

[5] Arima A, Harvey M and Shimizu K 1969 Phys. Lett. 30B 517

[6] Ginocchio J N 1997 Phys. Rev. Lett. 78436

[7] Ginocchio J N and Leviatan A 1998 Phys. Lett. B 4251

[8] Bohr A, Hamamoto I and Mottelson B R 1982 Phys. Scr. 26267

[9] Beuschel T, Blokhin A L and Draayer J P 1997 Nucl. Phys. A619 119

[10] Dudek J, Nazarewicz W, Szymanski Z and Leander G A 1987 Phys. Rev. Lett. 591405

[11] Nazarewicz W, Twin P J, Fallon P and Garrett J D 1990 Phys. Rev. Lett. 641654

[12] Stephens F S et al 1998 Phys. Rev. C57 R1565

[13] Mottelson B, 1991 Nucl. Phys. A522 1

[14] Blokhin A L, Bahri C and Draayer J P 1995 Phys. Rev. Lett. 744149

[15] Ginocchio J N and Madland D G 1998 Phys. Rev. C 571167

[16] Greiner W, Müller B and J. Rafelski 1985 Quantum Electrodynamics of Strong Fields (New York: Springer-Verlag)

[17] Bell J S and Ruegg H 1975 Nucl. Phys. B98 151 
[18] Serot B D and Walecka J D 1986 The Relativistic Nuclear Many - Body Problem in Advances in Nuclear Physics, edited by J. W. Negele and E. Vogt, Vol. 16 (New York: Plenum)

[19] Nikolaus B A, Hoch T and Madland D G 1992 Phys. Rev. C46 1757

[20] Cohen T D, Furnstahl R J, Griegel K and Jin X 1995 Prog. in Part. and Nucl. Phys. 35221

[21] Lalazissis G A, Gambhir Y K, Maharana J P, Warke C S and Ring P 1998 LANL archives nucl-th/9806009

[22] Meng J, Sugawara-Tanabe K, Yamaji S, Ring P and Arima A 1998 Phys. Rev. C58 R628

[23] Cheng T. P and Li L. F. 1984 Gauge Theory of Elementary Particle Physics (New York: Oxford University Press)

[24] Ginocchio J N, submitted to Phys Rev C; LANL archives nucl-th/9812025. 


\section{TABLES}

TABLE I. ${ }^{208} \mathrm{~Pb}$ pseudospin doublet energy splittings

\begin{tabular}{llll}
$\tilde{\ell}$ & ps doublets & $\epsilon_{\tilde{\ell}+1 / 2}-\epsilon_{\tilde{\ell}-1 / 2}(\mathrm{RMA})[15 n$ & $\epsilon_{\tilde{\ell}+1 / 2}-\epsilon_{\tilde{\ell}-1 / 2}(\mathrm{EXP})$ \\
\hline & neutrons & & 1.073 \\
4 & $0 h_{9 / 2}-1 f_{7 / 2}$ & 2.575 & -0.328 \\
2 & $1 f_{5 / 2}-2 p_{3 / 2}$ & 0.697 & \\
\hline & protons & & 1.791 \\
3 & $0 g_{9 / 2}-1 d_{5 / 2}$ & 4.333 & 0.351
\end{tabular}




\section{FIGURES}

FIG. 1. Examples of pseudospin doublets in the ${ }^{208} \mathrm{~Pb}$ region. $n_{r}$ is the radial quantum number of the state, $\ell$ is the orbital angular momentum, $j$ the total angular momentum.

FIG. 2. Pseudospin doublet energy splittings normalised by $2 \tilde{\ell}+1$ as a function of the average binding energy $\langle\epsilon\rangle . n_{r}$ is the radial quantum number of the state with the lower orbital quantum number

FIG. 3. Single - particle (s.p.) energies for neutron pseudospin partners as a function of deformation

FIG. 4. ${ }^{208} \mathrm{~Pb}$ lower component wavefunctions $f_{\tau, \tilde{\ell}-1 / 2}(\mathrm{r})$ (dash line), $-f_{\tau, \tilde{\ell}+1 / 2}(\mathrm{r})(\mathrm{dot}-$ dash line) for the $\left(2 s_{1 / 2}, 1 d_{3 / 2}\right)$ pseudospin doublet as a function of the radius $\mathrm{r}$

FIG. 5. ${ }^{208} \mathrm{~Pb}$ : a) energy spectrum; b,c,d) upper (g) and lower (f) components of the Dirac wavefunction for pseudospin doublets as a function of the radius $\mathrm{r}$ 


\section{Examples of Pseudospin Doublets}

$$
\begin{gathered}
\left(n_{r} \ell j ;\left(n_{r}-1\right) \ell+2, j+1\right) \\
\mathrm{j}=\tilde{\ell} \pm \tilde{s}, \tilde{s}=1 / 2 ;
\end{gathered}
$$

$\tilde{\ell}$ pseudo-orbital angular momentum, $\tilde{s}$ pseudo-spin
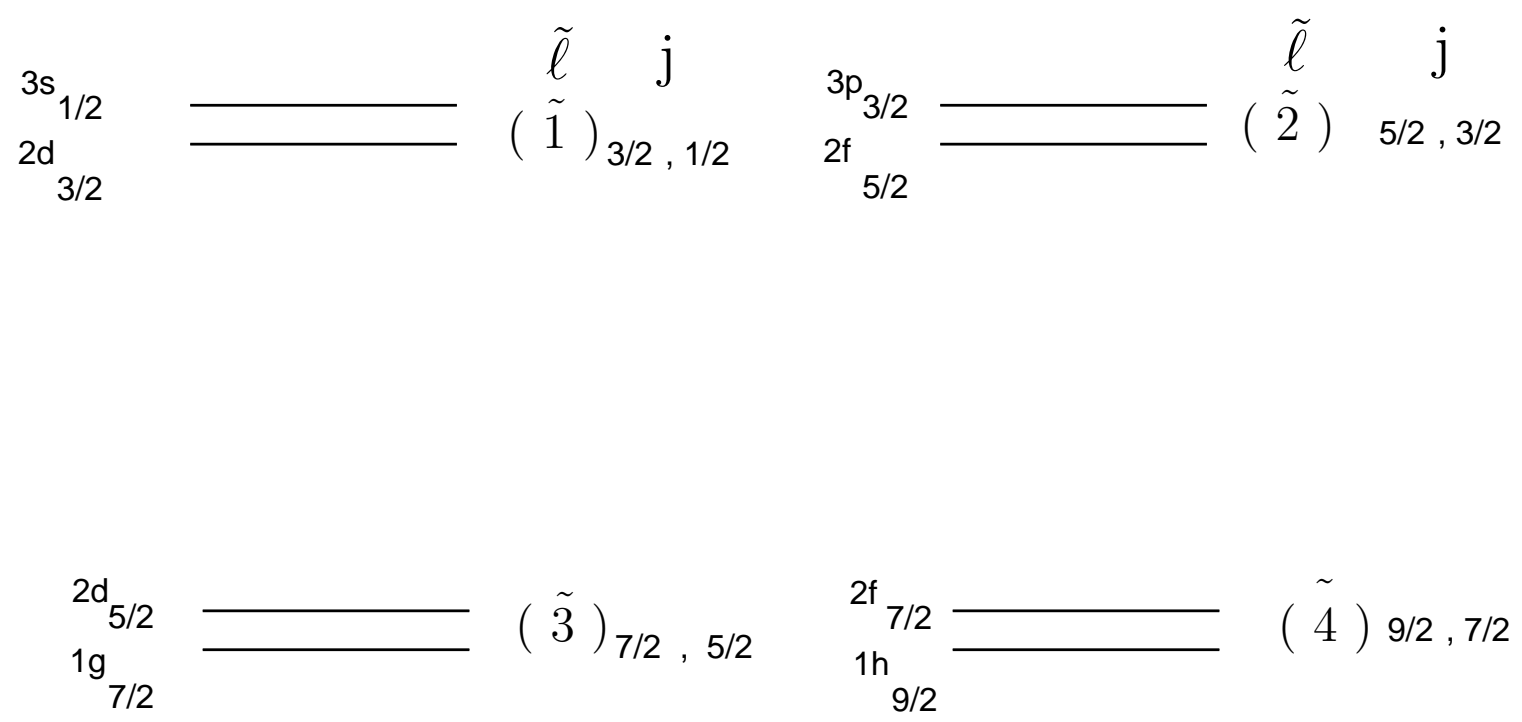


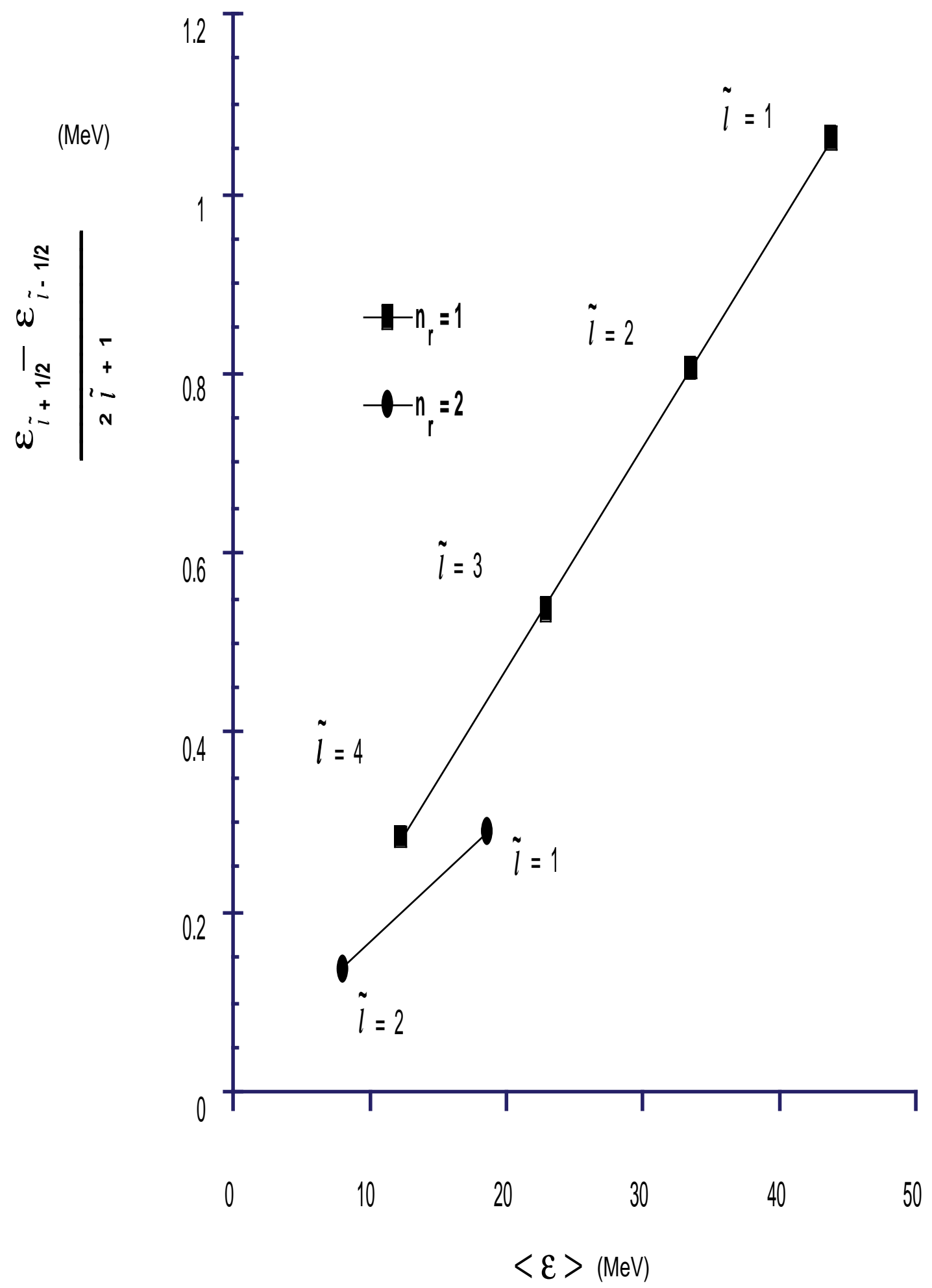




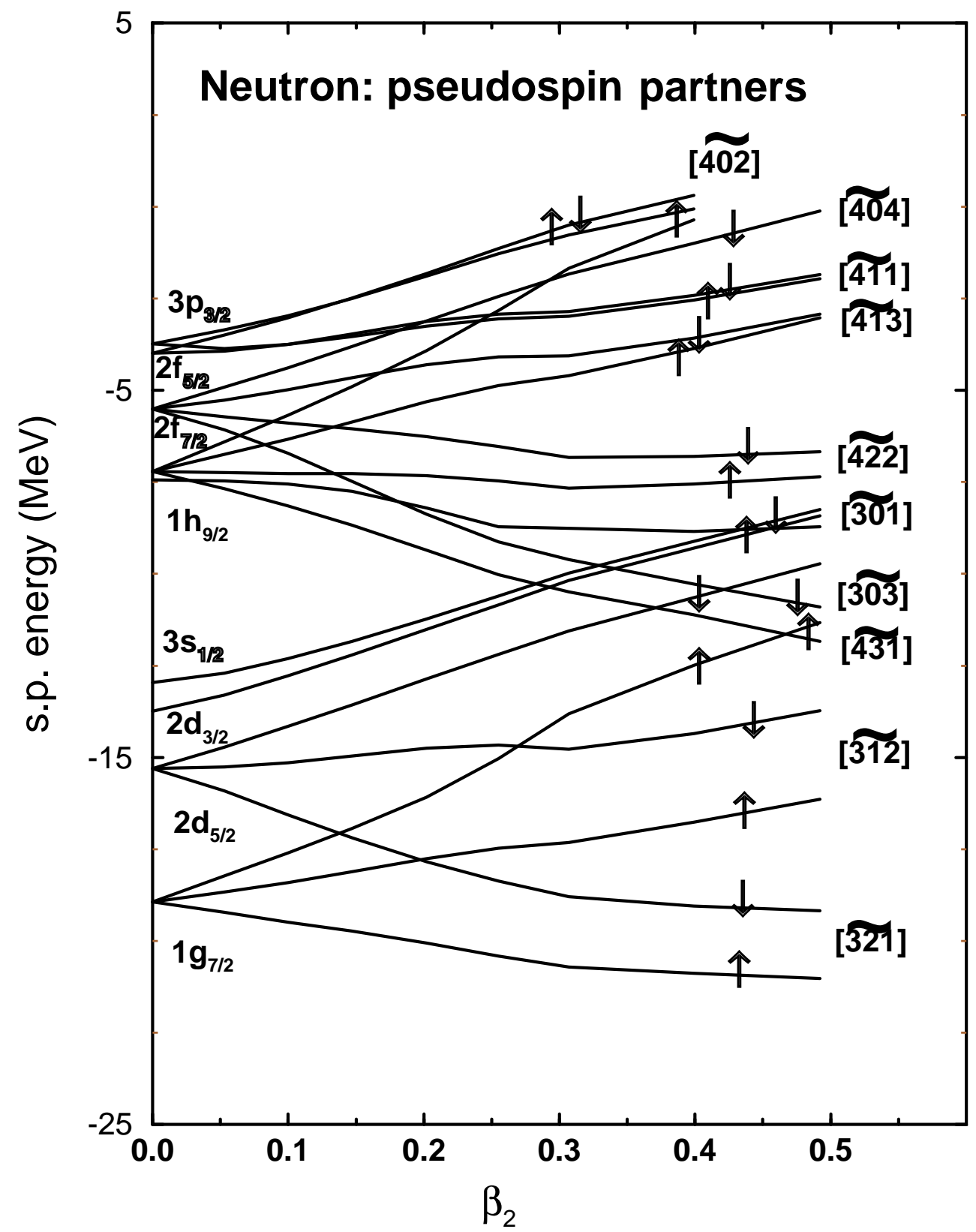




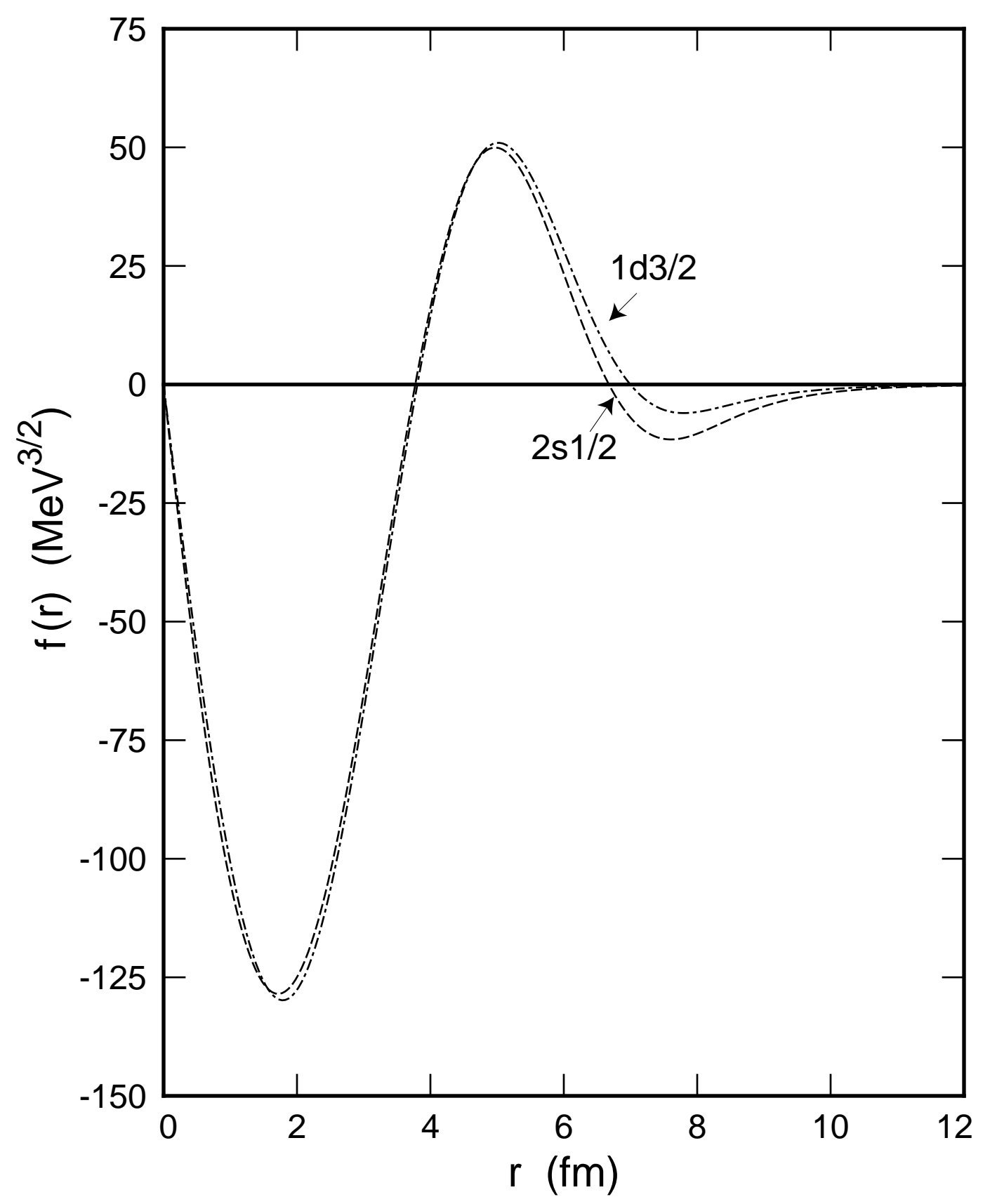



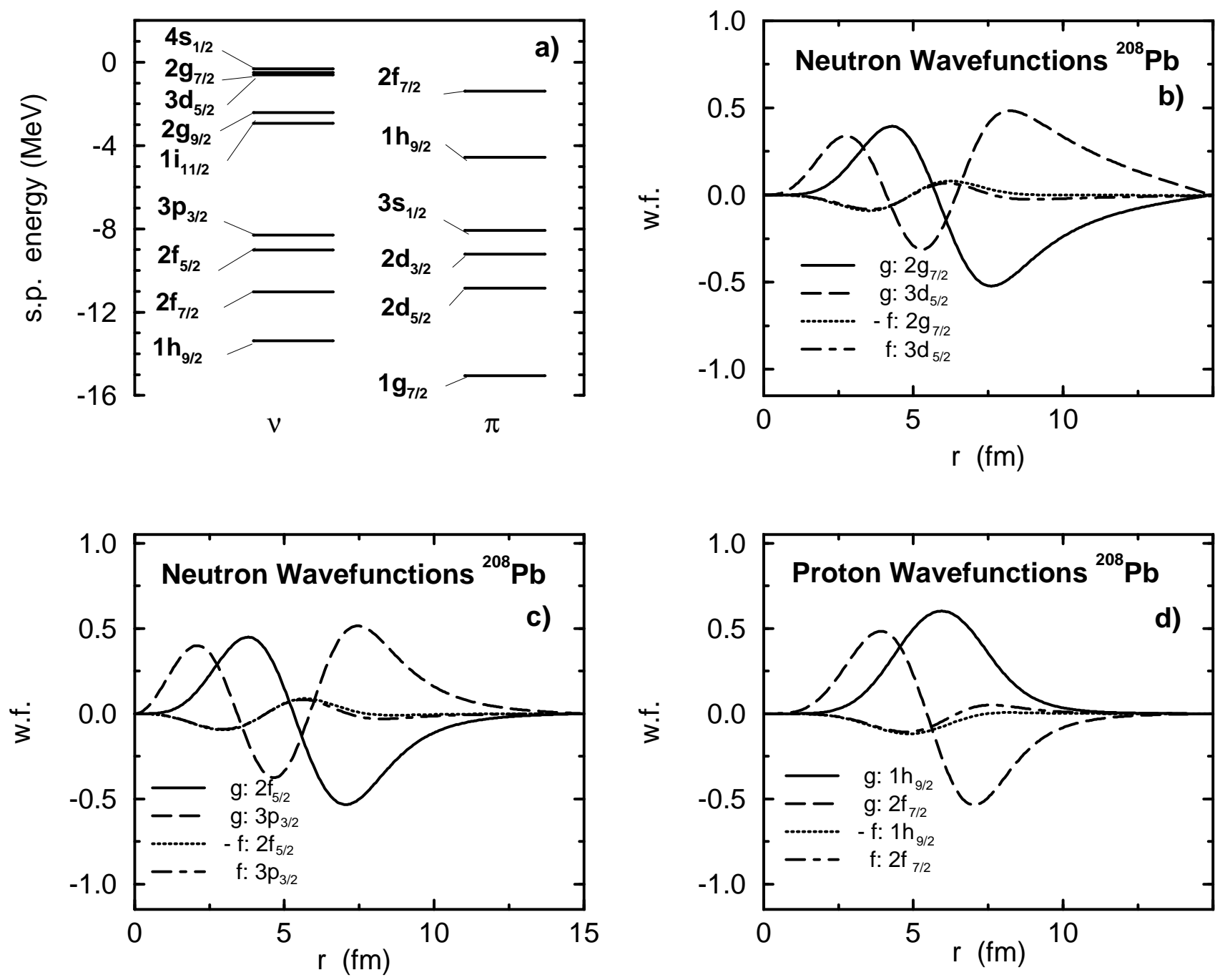